\title{
LETTERS
}

\section{Problems with hydromorphone prescribing as a response to the opioid crisis}

In January 2018, Dr. Tyndall advocated for prescribing hydromorphone as a response to the presence of illicitly manufactured fentanyl in the street opioid supply. ${ }^{1}$ Two years later, the opioid crisis has not abated. Between Jan. 1, 2018, and Mar. 31, 2019, 5670 Canadians died an apparent opioid-related death. ${ }^{2}$ Fentanyl continues to be pervasive in the illicit opioid supply. The scale of grief and loss for the families and communities of individuals represented in these statistics is horrifying. In response, some physicians have started prescribing hydromorphone tablets to people using illicit fentanyl with the intent to reduce the risk of exposure to this poisonous drug supply. ${ }^{3}$ Termed hydromorphone maintenance therapy (HMT), this practice entails hydromorphone being dispensed at a pharmacy at intervals of 1 to several days, all as take-home doses in the range of 16-24 tablets of $8 \mathrm{mg}$ daily. Most patients prescribed HMT will crush and inject the tablets. ${ }^{4}$ The practice has not been peer-reviewed.

Current accessible options to treat opioid use disorder for people using fentanyl are oral opioid agonist treatments (OAT) with buprenorphine, methadone, or slow-release oral morphine (SROM). For patients retained in these treatments, the benefits are proven; all-cause mortality is reduced, and health and social functioning are improved. ${ }^{5,6}$

For people who have not succeeded with oral OAT, supervised injectable opioid agonist treatment (SIOAT) has been proven safe and effective. ${ }^{7}$ It is intensive in terms of resources required, because health professionals prepare and oversee all patient selfadministration of injectable opioids. The injectable medications, diacetylmorphine or hydromorphone, are costly.

Practitioners and funders may think that, with HMT, they can reap all the health and social benefits of SIOAT without excess risks at a fraction of the cost. However, it is important to look at HMT through the lenses of safety, effective- ness, risks and potential adverse changes in the larger treatment ecosystem that, while intended to reduce risks of use of illicit fentanyl, could expose people using opioids and the public to additional risks.

Injecting crushed tablets compared with a pharmaceutical injectable preparation exposes a patient to risk of infection. It's impossible to make injected tablets as sterile as a medication intended for injection. If pharmacists prepare an injectable medication, the practice must be compliant with National Association of Pharmacy Regulatory Authorities standards. ${ }^{8}$

Compared with OAT with buprenorphine or methadone, take-home opioid maintenance programs expose patients to excess risks. In a French epidemiological study by Bertin and colleagues, compared with standard treatments, the group accessing take-home morphine had higher overall mortality, more overdoses and more infections. ${ }^{9}$ Overdoses are not limited to the person receiving the prescription. A study from the United States showed that opioid overdoses were almost 3 times more frequent in family members of people prescribed opioids for home use than in families where opioids were not prescribed. ${ }^{10}$

It's not easy for some people who inject opioids to let go of the feelings and rituals of injecting to go to an oral-only treatment. Defining features of opioid use disorder are an overwhelming craving/desire to use the substance that can eclipse other functional goals, loss of control over the use of opioids, and use despite harmful consequences (some related to criminalization and stigma, but many related to the opioid use and injection itself). ${ }^{11}$ People may choose to turn away from a proven safe treatment with oral medication, in favour of an unproven and riskier option. There are already signs of this happening in the communities where safer supply prescribing is practised (Dr. Sharon Koivu, London Health Sciences Centre, London, Ont.: personal communication). There can be real barriers to making traditional oral treatment like methadone work for patients; these barriers should be addressed before prescribing HMT.
The national clinical guideline on injectable opioid agonist treatment for opioid use disorder recommends that prescribers of SIOAT be well-versed in and provide oral OAT, including methadone. ${ }^{12}$ As SIOAT is positioned as part of a treatment continuum that includes oral options from more to less intensive, $20 \%$ $30 \%$ of people on SIOAT choose to deescalate their treatment to oral-only options in the first year. Few, if any, of the practitioners currently offering HMT prescribe methadone, and HMT is positioned as separate from the treatment system. This has the effect of ghettoizing patients and limiting their options. Patients engaged in HMT face unnecessary barriers if they have to go to a different doctor and location when they are ready to try methadone again.

Some may presume that HMT could completely displace illicit fentanyl use. Another possibility is that patients prescribed hydromorphone can sell tablets to buy fentanyl. This would have the effect of increasing the overall opioid burden in the community as well as the availability of hydromorphone for new initiates to opioid use. The national clinical guideline on SIOAT does not endorse any take-home doses of hydromorphone. ${ }^{8}$

With HMT positioned as a separate entity from the treatment continuum, there is no incentive for patients to deescalate their treatment, especially when a supply of hydromorphone tablets can be used to generate income. For patients who decide to use their hydromorphone supply this way, there is no simple way to monitor this, and no exit strategy. Diversion of hydromorphone tablets may also confound analysis of the benefits of programs that currently include provision of a basic income.

Proponents of HMT use the language of trauma and violence-informed care and patient engagement, with the message that people who use drugs have expertise to bring to the way they wish to receive care. It is fundamental that patients have a voice in their own care. The NAOMI trial ${ }^{7}$ and existing SIOAT programs consulted extensively with people who use drugs on 
design and implementation. However, if we frame patient preference as the only criterion for medical suitability of a treatment, it means that raising safety concerns can be construed as an attack on people who use drugs. The implication from HMT prescribers is that, as physicians, we should use our power and privilege to provide an alternative to the toxic drug supply, and that not to do so is to participate in opioid-related deaths and the oppression of people who use drugs.

However, as currently practised, HMT is a direct challenge to the fundamental principles of addiction medicine, where structure is applied to mitigate risks and harms of prescribing opioids to patients who use them in risky ways. The emergence of HMT is a result of the dismal failure of our federal and provincial governments to enact rational drug policy and fund an adequate response to the opioid crisis. Now physicians are faced with the impossible task of drug policy reform through prescribing.

The most medically rational response of physicians faced with the epidemic of opioid-related deaths is to work to lower barriers to proven oral OAT and ensure these treatments are effortless to access. Oral OAT should be available in every supervised consumption site for anyone who wants a dose, and SIOAT should be provided under supervision at drug con- sumption sites as an alternative to toxic fentanyl, so people have a choice to use a safer drug if they wish. This would require leadership from provincial and federal governments to expand access to supervised consumption sites and fund access to treatments there.

We must bring science to bear and be mindful of unintended consequences of our prescribing choices, especially in the case of opioids - a lesson we have learned at great cost.

\section{Lisa A. Bromley MD}

Physician, The Ottawa Hospital; Sandy Hill Community Health Centre, Ottawa, Ont.

- Cite as: CMAJ 2020 March 2;192:E219-20. doi: $10.1503 / \mathrm{cmaj} .74065$

\section{References}

1. Tyndall M. An emergency response to the opioid overdose crisis in Canada: a regulated opioid distribution program. CMAJ 2018;190:E35-6.

2. National report: apparent opioid-related deaths (September 2019). Ottawa: Public Health Agency of Canada; modified 2019 Sept. 25. Available: https://health-infobase.canada.ca/datalab/national -surveillance-opioid-mortality.html (accessed 2019 Oct. 30).

3. Izenberg D, Marwaha S. Safe supply: the debate around prescribing opioids to people who use drugs. Healthy Debate 2019 Aug. 15. Available: https://healthydebate.ca/2019/08/topic/safe-supply (accessed 2019 Oct. 30).

4. Hales J, Kolla G, Man T, et al. Emergency safer supply programs (ESSP): a harm reduction informed guiding document for primary care teams [draft].
October 2019. Available: https://docs.google.com/ document/d/1hXRmNaOTB60wbmNqhBi5P_xxktEZ nXFDWKMkJMu5ALM/edit\# (accessed 2019 Oct. 30).

5. CRISM national guideline for the clinical management of opioid use disorder. Canadian Research Initiative in Substance Misuse; 2018. Available: https://crism.ca/wp-content/uploads /2018/03/CRISM_NationalGuideline_OUD-ENG.pdf (accessed 2019 Nov. 9 2019).

6. Bruneau J, Ahamad K, Goyer M-È, et al.; CIHR Canadian Research Initiative in Substance Misuse. Management opioid use disorder: a national clinical practice guideline. CMAJ 2018;190:E247-57.

7. Oviedo-Joekes E, Brissette S, Marsh DC, et al. Diacetylmorphine versus methadone for the treatment of opioid addiction. N Engl J Med 2009;361:777-86.

8. Guidance for injectable opioid agonist treatment for opioid use disorder. Vancouver: British Columbia Centre on Substance Use; 2017. Available: www.bccsu.ca/wp-content/uploads/2017 /10/BC-iOAT-Guidelines-10.2017.pdf (accessed 2019 Oct. 30).

9. Bertin C, Delorme J, Riquelme M, et al. Risk assessment of using off-label morphine sulfate in a population-based retrospective cohort of opioid -dependent patients. Br J Clin Pharmacol 2019 Aug. 6 [Epub ahead of print]. doi: 10.1111/ bcp. 14082.

10. Khan NF, Bateman BT, Landon JE, et al. Association of opioid overdose with opioid prescriptions to family members. JAMA Intern Med 2019;179:1186-92.

11. Definition of addiction. Rockville (MD): American Society of Addiction Medicine; 2019. Available: www.asam.org/resources/definition-of-addiction (accessed 2019 Nov. 9).

12. Fairbairn N, Ross J, Trew K, et al. Injectable opioid agonist treatment for opioid use disorder: a national clinical guideline. CMAJ 2019;191:E1049-56.

Competing interests: Lisa Bromley has received honoraria from Indivior for speaking engagements. 